\title{
Dielectronic Recombination of Ground-State and Metastable $\mathrm{Li}^{+}$Ions
}

\author{
A. A. Saghiri, ${ }^{1}$ J. Linkemann, ${ }^{1}$ M. Schmitt, ${ }^{1}$ D. Schwalm,${ }^{1}$ A. Wolf,${ }^{1}$ T. Bartsch, ${ }^{2}$ A. Hoffknecht,${ }^{2}$ A. Müller, ${ }^{2}$ \\ W. G. Graham, ${ }^{3}$ A. D. Price, ${ }^{4}$ N. R. Badnell, ${ }^{4}$ T. W. Gorczyca, ${ }^{5}$ J. A. Tanis ${ }^{5}$ \\ ${ }^{1}$ Max-Planck-Institut für Kernphysik, Heidelberg, and Physikalisches Institut der Universität, D-69029 Heidelberg, Germany \\ ${ }^{2}$ Institut für Kernphysik, Strahlenzentrum der Justus-Liebig-Universität, D-35392 Giessen, Germany \\ ${ }^{3}$ Department of Pure and Applied Physics, Queen's University, Belfast BT7 1 NN, Northern Ireland \\ ${ }^{4}$ Department of Physics and Applied Physics, University of Strathclyde, Glasgow G4 0NG, United Kingdom \\ ${ }^{5}$ Department of Physics, Western Michigan University, Kalamazoo, Michigan 49008, USA
}

(July 19, 1999 - Submitted to Phys. Rev. A)

Dielectronic recombination has been investigated for $\Delta n=$ 1 resonances of ground-state $\mathrm{Li}^{+}\left(1 s^{2}\right)$ and for $\Delta n=0$ resonances of metastable $\mathrm{Li}^{+}\left(1 s 2 s^{3} S\right)$. The ground-state spectrum shows three prominent transitions between 53 and $64 \mathrm{eV}$, while the metastable spectrum exhibits many transitions with energies $<3.2 \mathrm{eV}$. Reasonably good agreement of R-matrix, $L S$ coupling calculations with the measured recombination rate coefficient is obtained. The time dependence of the recombination rate yields a radiative lifetime of $52.2 \pm 5.0$ s for the $2{ }^{3} S$ level of $\mathrm{Li}^{+}$.

PACS number(s): 34.80.Lx, 32.70.Cs, 32.80.Dz

In collisions between ions and electrons, recombination [1] can occur either by radiative recombination (RR) (inverse of the photoelectric effect), or by dielectronic recombination (DR). This process starts by the resonant capture of a free electron, mediated by its interaction with a bound electron (inverse of an Auger transition), and then proceeds by a radiative transition to a stable recombined system. Dielectronic recombination is of fundamental interest because it gives insight into electron correlation, and of applied interest for the understanding of astrophysical and laboratory plasmas. Extensive experimental studies providing significant tests of the theory [1] have been conducted for H-like, He-like, and Lilike ions heavier than about carbon. For such systems, perturbative techniques can be successfully used in theoretical calculations because the electron-electron interaction is relatively weak compared to the electron-nucleus interaction. In contrast, for lighter ions perturbative techniques cease to produce results at the same level of accuracy. Hence, high quality data for light systems are required to test the reliability of theoretical predictions.

For the lightest $\mathrm{H}$-like ion, $\mathrm{He}^{+}$, DR was studied previously by three groups 2 (1) with successively higher energy resolution, and through a consequent feedback between experiment and theory good mutual agreement could be obtained. For the lightest He-like ion, $\mathrm{Li}^{+}$, the two-electron configuration places additional constraints on the theory of RR and DR. Moreover, the existence of relatively long-lived metastable $1 s 2 s^{1} S$ and $1 s 2 s^{3} S$ states with natural lifetimes of about $0.5 \mathrm{~ms}$ and $50 \mathrm{~s}$, respectively, requires recombination for both the ground state and the metastable state (especially the long-lived triplet state) to be understood. To date, only lowresolution $\mathrm{DR}$ measurements for $\mathrm{Li}^{+}$have been carried out [5]; the integrated ground-state and metastable recombination rates could be extracted, but more detailed comparisons between experiment and theory for specific excited-state configurations were not possible.

Here we report a high-resolution investigation of DR in $\mathrm{Li}^{+}$. Individual intermediate-excited state configurations contributing to DR could be resolved for groundstate $\mathrm{Li}^{+}\left(1 s^{2}{ }^{1} S\right)$ ions, where $\mathrm{DR}$ occurs via $\Delta n=1$ resonances $\left(1 s^{2}+e \rightarrow 1 s 2 \ln ^{\prime} l^{\prime}\right)$ at relative electron-ion energies of $53-64 \mathrm{eV}$, as well as for metastable $\mathrm{Li}^{+}\left(1 s 2 s^{3} S\right)$ ions which give rise to $\Delta n=0$ resonances $(1 s 2 s+e \rightarrow$ $1 s 2 \ln ^{\prime} l^{\prime}$ ) below $3.2 \mathrm{eV}$. The metastable spectrum is found to be particularly rich with many closely-spaced transitions. The time dependence of DR from metastable ions was also used to determine the radiative lifetime of the $2^{3} S$ level. Moreover, RR has been observed for relative electron-ion energies close to zero.

At the Heidelberg ion-storage ring and electron cooler facility (TSR) a beam of $13.3 \mathrm{MeV}{ }^{7} \mathrm{Li}^{+}$ions was produced in a tandem accelerator and injected into the ring. Using multiturn stacking injection, which lasted about $0.1 \mathrm{~ms}$, a typical circulating ion current of $5 \mu \mathrm{A}$ was obtained. While stored, the ions passed through the collinear electron beam of the electron cooler. First, by matching the electron and ion velocities at a laboratory electron energy of $1.04 \mathrm{keV}$ ('cooling energy') the ion beam was phase-space cooled to low $(\sim 0.3 \mathrm{mrad})$ divergence and $\sim 10^{-4}$ relative longitudinal momentum spread within about $10 \mathrm{~s}$. Recombination of the $\mathrm{Li}^{+}$ions was then measured as a function of the energy $E$ corresponding to the average relative velocity between the electrons and the ions (referred to as relative energy) by detuning the laboratory electron energy from the cooling energy by a variable amount. In the measurements, the relative energy was alternately set (on a time scale of milliseconds) first to $E \approx 140 \mathrm{eV}$ to determine the background recombination rate, then to a desired value in the energy ranges of ground- or metastable-state recombination, and then back to the cooling energy.

Those ions which recombined to form neutral $\mathrm{Li}$ are no longer deflected by the storage ring dipole magnet following the electron cooler, and can thus be counted with a surface-barrier detector behind this magnet. Field ion- 
ization in the motional electric field experienced by the fast $\mathrm{Li}$ atoms in the dipole magnet introduces a principal quantum number cutoff [6] of $n_{c}=7$ so that atoms reaching the magnet in states with $n>n_{c}$ are not detected.

The energy resolution was determined by the electron temperatures of $k T_{\perp}=18 \mathrm{meV}$ and $k T_{\|}=0.2 \mathrm{meV}$ [], which resulted in a FWHM energy spread of $\sim 0.02 \mathrm{eV}$ in the electron-ion center-of-mass (c.m.) frame at $E \lesssim 0.2$ $\mathrm{eV}$, increasing to $\sim 0.4 \mathrm{eV}$ at $E=60 \mathrm{eV}$. The electron current was $35 \mathrm{~mA}$ in the lower and $60 \mathrm{~mA}$ in the upper energy range, respectively, corresponding to electron densities $n_{e}$ of 5 and $8 \times 10^{6} \mathrm{~cm}^{-3}$.

Recombination was measured in a time interval of 12 $70 \mathrm{~s}$ after each injection, the total cycle time being close to the $1 / e$ storage lifetime of $\sim 60 \mathrm{~s}$ determined by the ground-state stripping rate in the rest gas, $\lambda_{g}^{s}$. Measurements were repeated for successive injections over several hours. From earlier experiments [8] employing laser cooling of ${ }^{7} \mathrm{Li}^{+}$ions on the $2{ }^{3} S-2{ }^{3} P$ transition, a substantial initial fraction $f_{m}^{0} \approx 0.1-0.3$ of metastable $2^{3} S$ ions is known to exist in the TSR under these running conditions. The unknown initial fraction of metastable singlet $\left(2{ }^{1} S\right)$ ions is expected to have decayed by the time the data were recorded. The decay constant of the triplet $\left(2^{3} S\right)$ metastable $\mathrm{Li}^{+}$ions stored in the ring was $\sim(19 \mathrm{~s})^{-1}$, given mainly by the sum of the rest-gas stripping rate $\lambda_{m}^{s}$ and the radiative decay rate $\lambda_{m g}^{\gamma}$ of the metastable ions. Stripping of $2{ }^{3} S$ ions is expected [9] to occur faster than that of $1^{1} S$ ions because of the lower ionization potential of the triplet state. The energy region of metastable DR $(E=0-3.5 \mathrm{eV})$ was scanned in the two time intervals of $12-42$ and $54-70 \mathrm{~s}$ after injection, while the ground-state DR spectrum $(E=52-64 \mathrm{eV})$ was measured between $42-54 \mathrm{~s}$. Details concerning the determination of the relative energy and the c.m. electron energy distribution can be found in Ref. [10]. The accuracy of the relative-energy scale is estimated to be $\pm 0.02 \mathrm{eV}$ at $1 \mathrm{eV}$ and \pm 0.09 at $60 \mathrm{eV}$.

With the recombination rate coefficients $\alpha_{g}(E)$ and $\alpha_{m}(E)$ for ground and metastable ions, respectively, and the metastable ion fraction $f_{m}(t)$, the measured, background subtracted recombination rate is given by

$$
\begin{aligned}
R(E, t)= & \eta \gamma^{-2} \cdot N_{i}(t) \cdot n_{e} \\
& \times\left[\left(1-f_{m}(t)\right) \alpha_{g}(E)+f_{m}(t) \alpha_{m}(E)\right],
\end{aligned}
$$

where $\gamma=1.002$ is the Lorentz factor due to the relativistic transformation between the ion rest frame and the laboratory frame, and $\eta$ the ratio between the interaction length $(1.5 \mathrm{~m})$ and the ring circumference $(55.4 \mathrm{~m})$. The number of stored ions $N_{i}(t)$ was determined from the circulating beam current measured by a non-destructive current probe as well as by counting events at a beam profile monitor 11.

In addition to the energy scans, the time dependence of the recombination rate on selected DR peaks was measured, performing a sequence of fast scans over resonances at $E=0.14$ and $1.2 \mathrm{eV}$ (representing mainly metastable-ion DR) and $54 \mathrm{eV}$ (representing only ground-state DR); simultaneously the beam current signals from the current probe and the beam profile monitor as well as the count rates at $E=0$ and at $E=140$ $\mathrm{eV}$ were recorded. The scan sequence was repeated 25 times after each injection, covering storage times of 12 $75 \mathrm{~s}$. The integrated count rates were fitted jointly as functions of time by two-component exponential decay curves, varying the two decay constants common to all curves and appropriate separate amplitudes. Under the assumption (justified below) that collisional re-feeding of the metastable state by rest gas collisions can be neglected, the two resulting time constants are $\lambda_{1}=\lambda_{g}^{s}$ and $\lambda_{2}=\lambda_{m}^{s}+\lambda_{m g}^{q}+\lambda_{m g}^{\gamma}+\lambda_{m}^{e}$, where $\lambda_{m g}^{q}$ denotes the collisional quenching rate and $\lambda_{m}^{e}$ is the electron impact ionization rate 12 of metastable ions in the electron cooler occuring while the relative energy is above the $2{ }^{3} \mathrm{~S}$ ionization threshold (the same effect for ground-state ions is negligible). Measurements of the time variation at five residual gas pressures differing by factors of up to $\sim 3$ yielded for $\lambda_{1,2}$ the results shown in Fig. 1. As $\lambda_{g}^{s}, \lambda_{m}^{s}$, and $\lambda_{m g}^{q}$ all vary proportional to the rest gas pressure, $\left(\lambda_{m}^{s}+\lambda_{m g}^{q}\right) / \lambda_{g}^{s}$ can be deduced from the slope of the straight line through these data, while the rest-gas independent decay rate $\lambda_{m g}^{\gamma}+\lambda_{m}^{e}$ of the metastable state follows from linear extrapolation to $\lambda_{1}=0$. After subtraction of the estimated [12] electron impact ionization rate of $\lambda_{m}^{e}=0.003 \pm 0.001 \mathrm{~s}^{-1}$ the radiative decay rate of $\lambda_{m g}^{\gamma}=0.0191 \pm 0.0018 \mathrm{~s}^{-1}$ is obtained, which corresponds to a natural lifetime of $\tau_{M 1}=52.2 \pm 5.0 \mathrm{~s}$ ( $1 \sigma$ error) for the $2{ }^{3} S$ level. This result matches well the existing theoretical predictions 13.14 of 49.04-49.14 s as well as the only previous experimental result of $\tau_{M 1}=58.6 \pm 12.9 \mathrm{~s}$ (2 $\sigma$ error) [15]. The fact that the slope of $\lambda_{2}$ vs. $\lambda_{1}$ lies close to the expected ratio of the metastable and groundstate rest-gas stripping rates $(\sim 2[9])$ indicates that the collisional quenching rate was small.

After normalization to the ion number $N_{i}(t)$ the timedependent rate in the $54-\mathrm{eV}$ peak is proportional to $1-f_{m}(t)$ [see Eq. (1)], while the rates in the 0.14- and $1.2-\mathrm{eV}$ peaks dominantly vary as $f_{m}(t)$ with a small additional contribution from ground-state RR that could be estimated. Fits to the normalized rates in the $54-\mathrm{eV}$ peak yielded only rather inaccurate results for the initial metastable fraction $f_{m}(0)=f_{m}^{0}$, scattering between 0.1 and 0.4 , because the change of the ground-state fraction $1-f_{m}(t)$ during the measuring time was only slightly larger than the statistical counting errors. Corresponding fits to the low- $E$ peaks (focusing on the signal decay at longer storage times) allowed the rate of collisional re-feeding of the $2{ }^{3} S$ state to be estimated to be $<0.02$ of the ground-state stripping rate $\lambda_{g}^{s}$, justifying the assumption made above within the statistical error limits.

The rates $R(E, t)$ were converted to the experimental rate coefficients $\alpha_{g}(E)$ and $\alpha_{m}(E)$ using Eq. (11); appropriate rate equations together with the measured time constants $\lambda_{1,2}$ yielded the functional dependence $f_{m}(t)$. 
In the low energy range $(0-3.5 \mathrm{eV})$ the measured rates contain contributions to DR and to RR from metastable ions $\left[\alpha_{m}(E)\right]$ and to RR from ground-state ions $\left[\alpha_{g}(E)\right]$. They were extracted separately combining the data from the two storage time intervals in which this energy region was scanned. The experimental recombination rate coefficient $\alpha_{m}(E)$ for metastable $\mathrm{Li}^{+}$ions is shown in Fig. 22. Also shown is the theoretical rate coefficient (see below) for both DR and RR convoluted with the experimental electron velocity distribution. The intrinsic determination of the metastable fraction being too inaccurate, it was set to $f_{m}^{0}=0.19$, which yields the best agreement of the data between 0.25 and $0.35 \mathrm{eV}$ (see bottom part of figure) with the theoretical prediction. Thus, $f_{m}^{0}$ is adjusted to fit the calculated non-resonant ( $\mathrm{RR})$ rate of metastable $\mathrm{Li}^{+}$. Different values of $f_{m}^{0}$ would essentially scale the experimental result for $\alpha_{m}(E)$ as $\propto 1 / f_{m}^{0}$. The value of $f_{m}^{0}=0.19$ is compatible with the observed time dependences, as well as with the values estimated from earlier laser cooling experiments [8]. The systematic error in the normalization to the ion and electron beam intensity is estimated to $\pm 20 \%$; however, in view of the uncertainty in $f_{m}^{0}$ we attribute an overall systematic error of $\pm 50 \%$ to $\alpha_{m}(E)$. The resulting smooth ground-state $\mathrm{RR}$ rate coefficient $\alpha_{g}$ reaches a value of $(2 \pm 1) \times 10^{-12} \mathrm{~cm}^{3} \mathrm{~s}^{-1}$ at near-zero relative energy and drops to $(2 \pm 1) \times 10^{-13} \mathrm{~cm}^{3} \mathrm{~s}^{-1}$ at $E=0.3 \mathrm{eV}$.

In the high energy range $(52-65 \mathrm{eV})$ only $\Delta n=1 \mathrm{DR}$ of ground-state ions occurs, the contribution from the $\mathrm{RR}$ of metastable and ground-state ions being negligible. $\left(\Delta n=1 \mathrm{DR}\right.$ of metastable $\mathrm{Li}^{+}$ions, leading to triply excited $\mathrm{Li}$, lies in different energy regions not scanned in this experiment.) The DR rate coefficient $\alpha_{g}(E)$ for ground-state $\mathrm{Li}^{+}$ions is shown in Fig. 3. The uncertainty in $f_{m}^{0}$ here yields only a small contribution to the systematic error since the metastable fraction at the time of measurement is already down to $\lesssim 0.04$. Hence, a systematic error of $\pm 20 \%$ is estimated for $\alpha_{g}(E)$.

To obtain the theoretical rate coefficients shown in Figs. 2 and 3 unified photorecombination cross sections representing both $\mathrm{DR}$ and $\mathrm{RR}$ were computed in $L S$ coupling using a radiation damped R-matrix approach [16, 17. In fact, this case is one of the few where damping is negligible [18], and we obtained the same results by applying detailed balance to undamped R-matrix photoionization cross sections. To compare the calculations with experiment, contributions to DR where one of the electrons occupies a configuration with $n^{\prime}>n_{c}=7$ were excluded from the calculations. The $\Delta n=0$ DR spectrum of metastable $\mathrm{Li}^{+}$ions (Fig. 2) is dominated by $n^{\prime}$ manifolds converging to the $2^{3} S-2{ }^{1} P$ excitation threshold at $3.2 \mathrm{eV}$ [configurations $\left.1 s 2 p\left({ }^{1} P\right) n^{\prime} l^{\prime}\right]$. At low energies terms of the configuration $1 s 2 p\left({ }^{3} P\right) 3 l^{\prime}$ also appear. There is good agreement between theory and experiment up to $0.6 \mathrm{eV}$, although the theoretical line energies seem to be too high by $\sim 0.03 \mathrm{eV}$ and the double structure of the peak at $0.14 \mathrm{eV}$ is not reproduced. At higher energies the theory appears to overestimate the DR cross section significantly, but still yields a resonable representation of the general spectral shape.

It appears that with $n_{c}=7$ we do not account for the recombination rate observed above $3 \mathrm{eV}$. This is probably due to Li atoms formed with $n>n_{c}$, cascading to lower $n$ states during the time-of-flight $(0.3 \mu \mathrm{s})$ prior to the dipole magnets. Furthermore, the sharp cutoff $n_{c}$ represents only an approximate description of the field ionization process [6]. Particularly large discrepancies between theory and experiment occur above the $2{ }^{3} S$ $2^{3} \mathrm{P}$ excitation threshold $(2.28 \mathrm{eV})$. Here, states with high angular momentum $l^{\prime} \geq 3$ strongly contribute to the calculated DR rate, as demonstrated by eliminating these states from the calculation (lower, dashed curve in Fig. 2). Using this $l$ cutoff brings the calculated DR rate much closer in magnitude to the experiment. The possibility that Li atoms in high $l$ states (formed by radiative stabilization of high- $l$ resonances) suffer field ionization even below the cutoff $n_{c}$ can probably be ruled out. A possible experimental explanation for these discrepancies could arise from stray electric fields in the interaction region, estimated to be $\lesssim 3 \mathrm{~V} / \mathrm{cm}$; the DR rate above $\sim 1.9$ $\mathrm{eV}$ is reduced by autoionization into additional open continua $\left(1 s 2 s^{1} S, 1 s 2 p^{3} P\right)$ and, as remains to be verified in detail, high- $l$ resonances may assume larger autoionization rates than calculated through $l$-mixing in the stray electric fields.

Regarding the ground-state DR (Fig. 3) experiment and theory are in good agreement for the $1 s 2 l 2 l^{\prime}$ configurations near 54 and $56 \mathrm{eV}$. These terms are well known from ion-atom collision [19,20] and photoionization studies 21,22]. Along the $1 s 2 p\left({ }^{1} P\right) n^{\prime} l^{\prime}$ series one higher resonance, probably from $1 s 2 p\left({ }^{1} P\right) 3 l^{\prime}$, is observed near 60 $\mathrm{eV}$, but higher series members apparently are reduced by autoionization into the $1 s 2 l$ continua. Near $60 \mathrm{eV}$ the energy-integrated theoretical rate coefficient, representing several unresolved DR resonances, exceeds the experimental value (apparently a single resonance) by about a factor of 3. Also, the calculations predict a higher peak near $62 \mathrm{eV}$ due to Rydberg states with $n=4-7$.

In summary, $\mathrm{Li}^{+}$ions with a strong population of the long-lived $2{ }^{3} S$ state were stored for times on the order of the natural lifetime of the metastable level. This lifetime was determined with an error of $\pm 10 \%$, confirming the theoretical predictions. Experimental DR rate coefficients were obtained separately for the metastable and the ground state. DR from the $2^{3} S$ ions shows a rich spectrum of lines from doubly excited, higher angular momenta configurations of Li that cannot be reached by photoionization from the $\mathrm{Li}$ ground state and have not been observed before; their description presents a significant and important challenge to the theory of this fundamental two-electron system. An R-matrix calculation reproduces the spectral shape reasonably well. The ground-state DR spectrum shows three prominent and several weaker lines at energies of 54-62 eV, and there is reasonable agreement with theoretical calculations except for lines near the $1 s 2 l$ excitation thresholds 
$(>60 \mathrm{eV})$.

This work was supported by the Human Capital and Mobility Programme of the European Community, and by the German Ministry of Education, Science, Research, and Technology (BMBF) under contracts no. 06 HD 854I and 06 GI 848. J.A.T. was supported in part by the Division of Chemical Sciences, Office of Basic Energy Sciences, Office of Energy Research, U.S. Department of Energy.

[1] See Recombination of Atomic Ions, edited by W. G. Graham, W. Fritsch, Y. Hahn, and J. A. Tanis (Plenum Press, New York, 1992).

[2] J. A. Tanis et al., Nucl. Instrum. Methods B56/57, 337 (1991); R. R. Haar et al., Phys. Rev. A 47, R3472 (1993).

[3] T. Tanabe et al., Phys. Rev. A 45, 276 (1992).

[4] D. R. DeWitt et al., J. Phys. B 28, L147 (1995).

[5] P. A. Závodszky et al., Phys. Rev. A 58, 2001 (1998).

[6] E. P. Kanter et al., Phys. Rev. A 29, 583 (1984).

[7] S. Pastuszka et al., Nucl. Instrum. Methods A369, 11 (1996).

[8] S. Schröder et al., Phys. Rev. Lett. 64, 2901 (1990); R. Grieser et al., Hyp. Interact. 108, 241 (1997).

[9] V. S. Nikolaev, Sov. Phys. Usp. 8, 269 (1965) [Usp. Fiz. Nauk 85, 679 (1965)].

[10] G. Kilgus et al., Phys. Rev. A 46, 5730 (1992).

[11] B. Hochadel et al., Nucl. Instrum. Methods A343, 401 (1994).

[12] K. Berrington, J. Phys. B 31, 313 (1998).

[13] G. W. F. Drake, Phys. Rev. A 3, 908 (1971).

[14] A. Devrianko, I. M. Savukov, W. R. Johnson, and D. R. Plante, Phys. Rev. A 58, 4453 (1998), and references therein.

[15] R. D. Knight and M. H. Prior, Phys. Rev. A 21, 179 (1980).

[16] F. Robicheaux, T. W. Gorczyca, M. S. Pindzola, and N. R. Badnell, Phys. Rev. A 52, 1319 (1995).

[17] T. W. Gorczyca and N. R. Badnell, J. Phys. B 29, L283 (1996).

[18] N. R. Badnell, T. W. Gorczyca and A. D. Price, J. Phys. B 31, L239 (1998).

[19] P. Ziem, R. Bruch, and N. Stolterfoht, J. Phys. B 8, L480 (1976).

[20] M. Rødbro, R. Bruch, and P. Bisgaard, J. Phys. B 12, 2413 (1979).

[21] L. M. Kiernan et al., Phys. Rev. Lett. 72, 2359 (1994).

[22] L. M. Kiernan et al., J. Phys. B 28, L161 (1995).

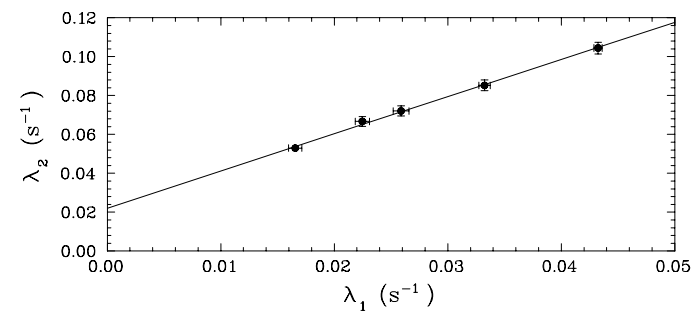

FIG. 1. Decay constants representing the total decay rates $\lambda_{1}$ of ground-state and $\lambda_{2}$ of metastable $\mathrm{Li}^{+}$ions, as measured for five residual gas pressures. Extrapolation to $\lambda_{1}=0$ (corresponding to vanishing pressure) yields $\lambda_{m g}^{\gamma}+\lambda_{m}^{e}=0.0221 \pm 0.0015 \mathrm{~s}^{-1}$

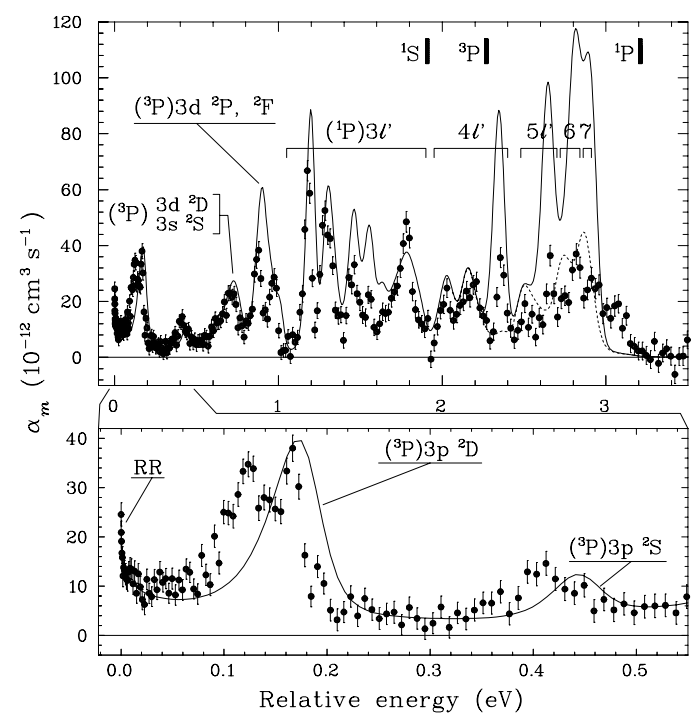

FIG. 2. Recombination rate coefficient $\alpha_{m}(E)$ for metastable $\mathrm{Li}^{+}\left(1 s 2 s^{3} S\right)$ ions. Dots: experimental data, obtained assuming an initial metastable fraction of $f_{m}^{0}=0.19$. Full line: theory for $n_{c}=7$; labels refer to configurations $1 s 2 p\left({ }^{1,3} P\right) n^{\prime} l^{\prime}$ and to excitation thresholds from the $2^{3} S$ state. Dashed line: theory omitting all states with $l^{\prime} \geq 3$.

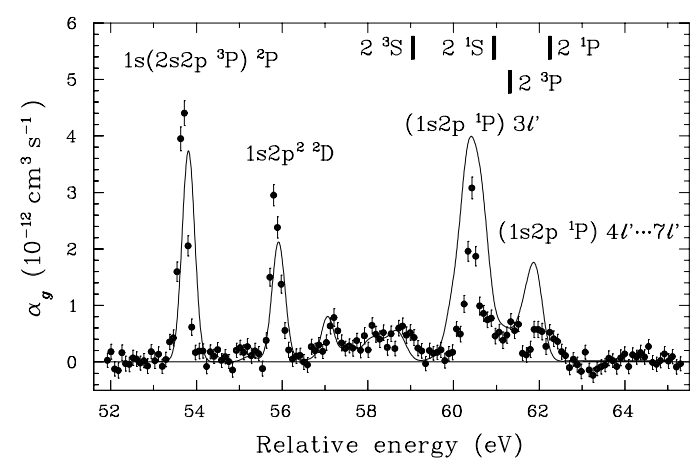

FIG. 3. Dielectronic recombination rate coefficient $\alpha_{g}(E)$ for ground-state $\mathrm{Li}^{+}\left(1 s^{2}\right)$ ions. Dots: experimental data; line: theory for $n_{c}=7$. Excitation thresholds from the $1^{1} S$ state are indicated. 